\title{
Acido urico, spettatore o artefice dell'aumentato rischio cardiovascolare e renale?
}

\author{
Francesca Viazzi, Giovanna Leoncini, Roberto Pontremoli
}

Dipartimento di Cardionefrologia, Università di Genova, IRCCS Azienda Ospedaliera Universitaria San Martino-

IST, Istituto Nazionale per la ricerca sul Cancro, Genova

\begin{abstract}
URIC ACID: BYSTANDER OR ACTIVE PLAYER IN THE DEVELOPMENT OF CARDIOVASCULAR AND RENAL DAMAGE?
Abstract. A number of epidemiological studies have reported an association between serum uric acid (SUA) levels and a wide variety of high-risk conditions including hypertension, insulin resistance, kidney and cerebro-cardiovascular diseases. Moreover, the sharp rise in the prevalence of these conditions has also been associated with a progressive rise in SUA levels in the general population (from less than $3.5 \mathrm{mg} / \mathrm{dL}$ in the $1920 \mathrm{~s}$ to $6.5 \mathrm{mg} / \mathrm{dL}$ in the 1970s). In this article we critically analyze whether SUA plays an active role in the development of atherosclerotic damage or should be considered an innocent bystander. Although the treatment of asymptomatic hyperuricemia is not yet recommended for the primary prevention of cardiovascular diseases, recent studies suggest a direct relationship between pharmacological reduction of SUA and cardiovascular and renal protection.
\end{abstract}

Key words: Uric acid, Organ damage, Chronic kidney disease, Cardiovascular risk

Conflict of interest: None.

Ricevuto: 11 Dicembre, 2012; Accettato: 3 Febbraio 2013

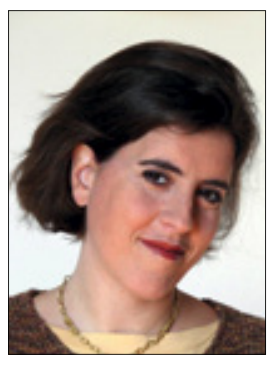

Francesca Viazzi

\section{Iperuricemia ed epidemiologia} del rischio $\mathrm{CV}$ e renale

La gotta rappresenta la più comune forma di artrite nell'uomo e la sua prevalenza sta progressivamente crescendo nelle ultime decadi. Vi sono forti evidenze che legano gotta e aumentati livelli di acido urico (AU) a condizioni cliniche a elevato rischio cardiovascolare quali ipertensione, insulino-resistenza e danno cerebro-cardiovascolare (1) e renale (2).

La prima osservazione di un'associazione positiva tra AU e ipertensione risale a più di un secolo fa (3). In uno studio multicentrico condotto su 2145 ipertesi taiwanesi, la presenza di iperuricemia era 1.5 volte più alta negli uomini e 1.7 volte più alta nelle donne rispetto alla popolazione generale (4). Nonostante la relazione tra $\mathrm{AU}$ e valori pressori sia complicata da numerosi fattori confondenti quali età, diabete, obesità, assunzione di alcool o di sale e stato dei volumi, i dati che descrivono questa relazione sono consistenti in numerosi studi. Inoltre, una recente metanalisi su studi prospettici indica che i pazienti iperuricemici presentano un rischio maggiore di sviluppo fu- turo di ipertensione, indipendentemente dai comuni fattori di rischio. Tale relazione è più forte nei giovani e nelle donne (5). Studi sperimentali condotti sia su animali che nell'uomo suggeriscono l'esistenza di due step nei meccanismi patogenetici che legano l'AU allo sviluppo dell'ipertensione (6). Inizialmente l'AU attiva il Sistema Renina Angiotensina Aldosterone (SRAA) e sopprime l'ossido nitrico (ON), portando a un aumento delle resistenze vascolari a livello sistemico. A questo seguono lo sviluppo di arteriosclerosi dell'arteriola afferente al glomerulo renale e l'instaurarsi di sodio-ritenzione. Inoltre, in due studi randomizzati controllati, viene descritto come la terapia con allopurinolo o probenecid sia in grado di normalizzare la pressione in adolescenti iperuricemici ipertesi $(7,8)$. Sebbene questi dati supportino un ruolo patogenetico dell'acido urico nello sviluppo di ipertensione in questo specifico contesto demografico, rimane da confermare l'applicabilità di questi risultati a una popolazione adulta.

Il fatto che la prevalenza della Sindrome Metabolica (SM) e di ciascuna delle sue componenti (9) aumenti in maniera progressiva all'aumentare dei livelli di AU ha indotto alcuni Autori a proporre che l'iperuricemia venisse inclusa nella definizione della SM. Inoltre, è stato ipotizzato che la presenza di valori elevati di $\mathrm{AU}$ in corso di SM non potesse essere interpretata 
come un puro effetto dell'insulino-resistenza. Viceversa, l'AU potrebbe giocare un ruolo determinante nella patogenesi della SM poiché, grazie alla sua capacità di ridurre la liberazione di ossido nitrico $(\mathrm{ON})$, promuove lo sviluppo della disfunzione endoteliale. I livelli sierici di AU hanno inoltre dimostrato di predire lo sviluppo di SM nell'uomo, correlandosi in maniera diretta con i valori di glicemia nella popolazione sana. Questa relazione non si è invece confermata nei pazienti diabetici, nei quali a stati iperglicemici corrispondono bassi livelli di acido urico. Una recente metanalisi che descrive la relazione tra livelli di AU e rischio di sviluppare diabete nella popolazione generale riporta come ad ogni aumento di $1 \mathrm{mg} / \mathrm{dL}$ di AU corrisponda un rischio di sviluppare diabete di tipo 2 aumentato del 17\% (10). Più recentemente, la capacità dell'AU di predire lo sviluppo futuro del diabete è stata descritta in diversi setting clinici: dai soggetti normali agli ipertesi sia ad alto che a relativamente basso rischio (11) (Fig. 1). È interessante notare come i ratti sottoposti a una dieta ricca di fruttosio diventino non solo iperuricemici, ma sviluppino anche ipertensione, ipertrigliceridemia, iperinsulinemia, ipertensione glomerulare, vasocostrizione delle arteriole corticali e aterosclerosi dell'arteriola preglomerulare (12). Il fruttosio induce iperuricemia stimolando la conversione dell'ATP epatica ad ADP tramite la fruttochinasi; al consumo dell'ATP corrisponde un accumulo di AMP che, attivando l'AMP deaminasi, porta alla sintesi di AU. Per quanto concerne l'uomo, un'analisi epidemiologica ha sottolineato come l'assunzione di bevande dolcificate, la maggiore fonte di fruttosio nella nostra dieta, porti all'aumento dei livelli di AU e si associ a un rischio aumentato di gotta, ipertensione e diabete.

\section{Acido urico e meccanismi di patologia CV}

Numerosi studi sperimentali suggeriscono diversi meccanismi patogenetici attraverso i quali l'AU sarebbe in grado di promuovere il danno aterosclerotico. Johnson et al. (13) hanno avuto il merito di sviluppare un modello di ratti resi moderatamente ipertesi mediante la somministrazione di Acido Oxonico che, inibendo l'enzima uricasi, rende i ratti incapaci di metabolizzare l'AU ad allantoina e, quindi, più simili all'uomo (che, nel processo evolutivo, è rimasto privo di questo enzima). I ratti così trattati non solo diventano iperuricemici, ma sviluppano anche ipertensione con un meccanismo legato da un lato allo sviluppo di arteriolosclerosi con inibizione della liberazione di Ossido Nitrico $(\mathrm{ON})$ e attivazione del Sistema Renina Angiotensina Aldosterone e, dall'altro, allo sviluppo di sodio-sensibilità. Inoltre, l'AU è in grado di stimolare le cellule muscolari lisce vascolari del ratto alla liberazione di monocyte chemoattractant protein-1 (MCP-1) (14) che, a sua volta, richiama i macrofagi a infiltrare la parete dei vasi aterosclerotici.

È stato ipotizzato che l'aumento dell'AU in pazienti con malattia aterosclerotica dei vasi possa riflettere un'aumentata generazione di AU causata da ischemia tissutale, stress ossidativo o eccessiva assunzione di alcool o fruttosio. Tuttavia, potrebbe anche essere il risultato di una sua ridotta escrezione renale imputabile al concomitare di ridotto filtrato glomerulare, iperinsulinemia, vasocostrizione e uso di diuretici.

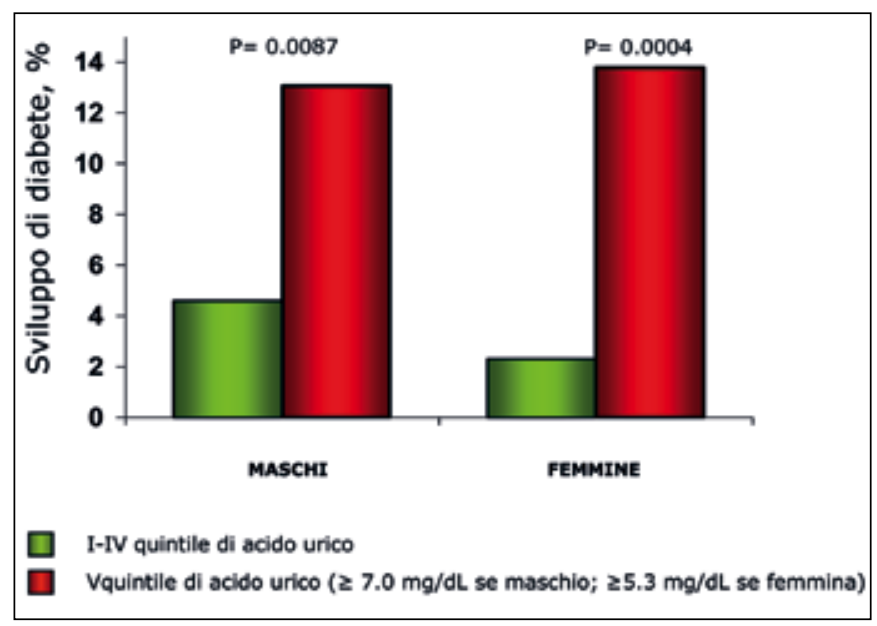

Fig. 1 - Aumentati livelli di acido urico si associano a un aumentato rischio di sviluppare diabete in un gruppo di ipertesi essenziali $(n=758)$ seguiti per un follow-up medio di 11 anni (10).

Nonostante alcuni studi non trovino una correlazione stretta tra AU e danno d'organo (15), la maggior parte dei dati disponibili in letteratura riporta una forte e indipendente associazione tra livelli di AU e segni sub-clinici di aterosclerosi quali ispessimento miointimale a livello carotideo (16), stenosi dell'arteria renale e arteriopatia periferica.

Tuttle è stata la prima Autrice a descrivere, in un gruppo di pazienti sottoposti a coronarografia (16), uno stretto legame tra incrementi di AU e arteriopatia coronarica (CAD). Successivamente, l'associazione tra iperuricemia e calcificazioni coronariche (CAC) è stata confermata in una serie di studi condotti su popolazioni a rischio perché caratterizzate dalla presenza di diabete di tipo 1, ipertensione o SM. Più recentemente, Krishnan (18) ha descritto un legame tra la presenza e la severità delle CAC e la concentrazione di AU in giovani adulti sani, confermando l'ipotesi che aumentati valori di AU possano essere coinvolti nel processo aterosclerotico, indipendentemente da altri fattori di rischio convenzionali.

I livelli di AU sono risultati correlati anche all'ipertrofia ventricolare sinistra (IVS) sia negli ipertesi (16) che in uomini giapponesi apparentemente sani. Iwashima et al. hanno dimostrato che l'AU è associato all'indice di massa ventricolare sinistra (LVMI) in maniera indipendente e hanno proposto la presenza simultanea di IVS e iperuricemia come un potente predittore di malattia CV (19). La relazione tra AU e massa ventricolare sinistra potrebbe essere la conseguenza della tendenza dell'iperuricemia ad associarsi ad altre condizioni cliniche ad alto rischio quali la disfunzione renale, l'ipertensione e l'obesità. Inoltre, l'AU potrebbe riflettere la produzione di superossido e di uno stato di stress ossidativo legato all'attivazione della xantinossidasi. Alternativamente, l'IVS potrebbe essere attribuibile, almeno in parte, alla stimolazione della disfunzione endoteliale, della proliferazione delle cellule muscolari lisce vascolari e dell'infiammazione dovuta proprio agli aumentati livelli di AU.

I pazienti con iperuricemia mostrano un'aumentata incidenza 
di eventi e morti per cause $C V(20,21)$, principalmente nelle popolazioni a più alto rischio. Nello studio LIFE (22), gli eventi CV erano correlati ai livelli di acido urico, anche se, dopo la correzione per la stratificazione del rischio secondo Framingham, tale relazione persisteva solo nelle donne. Risultati simili, che evidenziano un maggiore ruolo predittivo dell'AU nelle donne, derivano dalle analisi condotte su 4300 soggetti dello studio Rotterdam (23) e sulla popolazione del NHANES I (24).

Un'associazione indipendente tra infarto cardiaco e gotta o iperuricemia è stata descritta nello studio MRFIT condotto su 12866 uomini senza pregressa storia di cardiopatia ischemica (CHD), seguiti per 6.5 anni (25). Due recenti metanalisi condotte su studi prospettici mostrano una significativa e indipendente relazione tra iperuricemia anche lieve e sviluppo di CHD (HR 1.09) e morte cardiaca (HR 1.16) da un lato (26) e incidenza di stroke (HR 1.47) e morte cerebrovascolare (HR 1.26) dall'altro (27) (Fig. 2).

Indubbiamente, molti studi non hanno tenuto conto del ruolo confondente della disfunzione renale, un ben noto fattore di rischio per lo sviluppo di eventi CV $(24,26,28)$. Questo potrebbe aver indotto a sovrastimare la relazione tra iperuricemia e incidenti CV. Malgrado ciò, in numerosi studi più recenti, $\mathrm{i}$ livelli di acido urico sono risultati associati con la morbilità $\mathrm{CV}$ anche dopo aver corretto per la funzione renale (29). Nei pazienti in emodialisi, l'interpretazione dei dati viene resa più complessa dal fatto che valori più bassi di acido urico sono caratteristici di uno stato di malnutrizione che condiziona, a sua volta, la mortalità $\mathrm{CV}$ e per tutte le cause $(28,30)$ Nello studio di Neri et al. (29), la correlazione tra AU e malattia CV era maggiore nei pazienti con malattia renale cronica (MRC) avanzata. Sebbene questo dato sia dibattuto, viene confermato in un sottogruppo di 839 pazienti arruolati nel trial MDRD e in altri studi condotti su pazienti portatori di trapianto renale. Infine, nonostante sia stato suggerito un ruolo attivo dell'iperuricemia nella perdita del rene trapiantato, aumentati livelli di acido urico non sembrano essere un fattore di rischio addizionale allo score di Framingham nel predire l'infarto cardiaco, la rivascolarizzazione coronarica o la morte cardiaca nei riceventi di trapianto di rene.

\section{Acido urico e sviluppo di malattia renale}

Dato che la concentrazione dell'AU è in funzione dell'equilibrio tra il catabolismo delle purine e l'escrezione di AU, la malattia renale può causare iperuricemia. In particolare, nell'insufficienza renale si assiste a una riduzione della filtrazione dei sali di urato; 1'acidosi, spesso concomitante, riduce la secrezione tubulare di AU; nel corso della terapia diuretica la frequente comparsa di iperuricemia è imputabile a un aumentato riassorbimento di AU ad opera del nefrone (31). L'associazione tra artrite gottosa e malattia renale è storicamente ben documentata. La nefropatia uratica è caratterizzata da depositi di cristalli di urato che stimolano fibrosi interstiziale e arteriolosclerosi. Malgrado ciò, i depositi di cristalli di urato non sono molto comuni nei pazienti gottosi sottoposti a biopsia renale, mentre sono molto più frequenti arteriolosclerosi e nefroangiosclerosi. Anche un aumento solo moderato

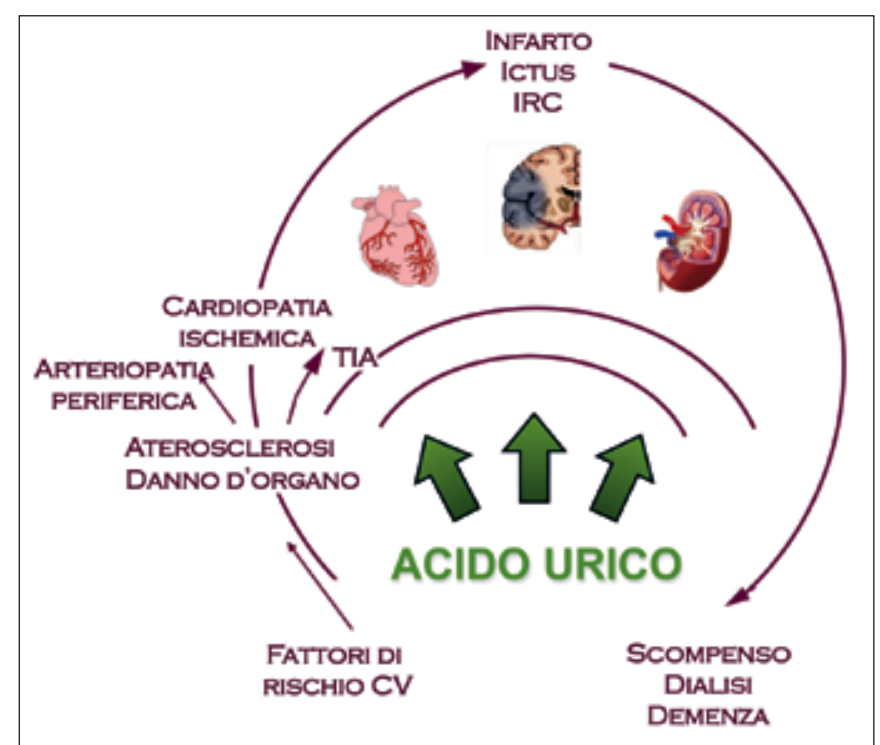

Fig. 2 - Acido urico e il continuum cardio-renale.

dei livelli di AU può associarsi a segni subclinici di sofferenza renale indagati con ecografia e Doppler (32).

È stata descritta anche una relazione positiva tra valori di AU e livelli di escrezione urinaria di albumina in pazienti con diabete di tipo 2 e ipertensione $(15,16)$ e in un'ampia coorte di soggetti preipertesi con funzione renale nella norma. Infine, $i$ livelli di AU sono linearmente associati alla severità dell'atrofia tubulare osservata in biopsie di pazienti con nefropatia a depositi di IgA. In accordo con questi risultati, in un modello animale la presenza di iperuricemia si è mostrata in grado di accelerare la progressione della malattia renale grazie all'aumento dei valori pressori ad essa associato e all'attivazione della cascata ciclossigenasica che, essendo implicata nello sviluppo della disfunzione endoteliale e nella produzione di trombossano, contribuisce all'aterosclerosi glomerulare. Tenendo conto di queste osservazioni, non stupisce che l'iperuricemia si associ in maniera indipendente a un aumentato rischio di presentare malattia renale cronica (MRC), come risulta da numerosi studi cross sectional condotti in Europa, Asia, USA e Cina sia nella popolazione generale che negli ipertesi (31). Nonostante questa evidenza, aumentati livelli di AU non vengono ancora universalmente considerati un vero e proprio fattore di rischio per la MRC. Infatti, frequentemente concomitano più tradizionali fattori di rischio quali diabete, ipertensione, dislipidemia, obesità, abitudine al fumo e insulino-resistenza che possono giustificarne l'insorgenza.

L'AU può avere un effetto tossico diretto sul rene. Inoltre, potrebbe essere il fattore intermedio che lega la presenza di ipertensione al danno renale poiché, causando sia vasocostrizione renale sia un aumento della pressione arteriosa sistemica, determina, di fatto, una progressione della MRC. Studi condotti allo scopo di valutare se l'AU possa essere considerato un fattore di rischio indipendente per lo sviluppo della malattia renale hanno fornito dati contrastanti (33).

Ishani et al. (33), esaminando un lungo follow-up dello studio 
MRFIT, hanno descritto descrivono un'associazione diretta tra AU e sviluppo di malattia renale terminale, anche se tale associazione si attenuava con l'esclusione dei pazienti con preesistente MRC (stima del filtrato glomerulare $<60 \mathrm{~mL} / \mathrm{min}$ o proteinuria).

Mentre questi risultati suggeriscono che l'AU possa essere un marker di ridotta funzione renale anziché una causa diretta di malattia renale incipiente, recentemente sono state pubblicate delle forti evidenze a favore dell'ipotesi che elevati valori di AU possano contribuire direttamente allo sviluppo della malattia renale. Uno studio condotto su 21475 soggetti sani seguiti per un follow-up medio di 7 anni ha analizzato la relazione tra livelli di acido urico e sviluppo di danno renale. Gli Autori concludono che livelli anche lievemente aumentati di AU sono in grado di aumentare del $26 \%$ il rischio di sviluppare una malattia renale de novo, anche tenendo conto di numerosi fattori di rischio confondenti, inclusa la stima del filtrato glomerulare al basale (34). Inoltre, negli studi ARIC e CHS, condotti su più di 13000 partecipanti con funzione renale normale, il modello statistico più complesso e sfavorevole mostrava un rischio di sviluppare danno renale aumentato del 7\% per ogni aumento di $1 \mathrm{mg} / \mathrm{dL}$ dei livelli di AU al basale (35). Tutto considerato, l'AU potrebbe indurre la malattia renale sia direttamente che indirettamente, favorendo altri noti meccanismi di danno.

L'iperuricemia è una complicanza abituale in pazienti portatori di trapianto di rene e dati retrospettivi suggerirebbero un suo più frequente sviluppo con l'utilizzo di prednisone e ciclosporina rispetto al tacrolimus (36). Una recente metanalisi condotta su 12 studi conclude che la presenza di iperuricemia (definita come acido urico $\geq 6.5 \mathrm{mg} / \mathrm{dL}$ nella donna e $\geq 7.0 \mathrm{mg} / \mathrm{dL}$ nell'uomo) è un fattore di rischio indipendente sia per lo sviluppo di nefropatia del trapianto (HR 1.65, 95\% CI 1.02-2.65) sia per la perdita del trapianto stesso (HR 2.01, 95\% CI 1.39-2.94) (37).

\section{Acido urico quale potenziale target della terapia cardio-renale}

Sebbene il ruolo patogenetico dell'AU nello sviluppo degli eventi $\mathrm{CV}$ e renali sia oggetto di numerose pubblicazioni, al momento attuale solo piccoli studi con ridotta potenza statistica hanno indagato circa l'effetto di una riduzione dell'AU secondaria a un intervento farmacologico. Questi, peraltro, suggeriscono il raggiungimento di un migliore controllo pressorio e di un rallentamento della progressione renale imputabile all'assunzione di terapie ipouricemizzanti (38). In una popolazione di 9924 veterani iperuricemici, l'impiego di allopurinolo, la molecola più usata in tale condizione clinica, si è associato a una riduzione del $25 \%$ della mortalità per tutte le cause (39).

Studi randomizzati mostrano, su piccole casistiche, riduzione dei valori pressori $(7,8)$, del danno $\mathrm{CV}$ e della velocità di progressione della MRC nei pazienti trattati con allopurinolo. Anche nello studio di Goicoechea (40), i pazienti sottoposti a tale trattamento per un adeguato follow-up di 24 mesi si giovavano di una ridotta progressione della MRC, di una ridotta infiammazione subclinica e di un minor rischio di ospedalizzazione e di eventi CV.

Rimane da chiarire se questi effetti, indubbiamente positivi, siano totalmente da ricondurre alla riduzione dell'AU o, al- ternativamente, al meccanismo d'azione del farmaco che, inibendo la xantinossidasi, è in grado di migliorare la disfunzione endoteliale.

Nello studio RENAAL, condotto su nefropatici con diabete di tipo 2 , i pazienti in trattamento con losartan avevano valori più bassi di $\mathrm{AU}$ e un minor rischio di eventi renali rispetto a quelli in placebo. In un'analisi post hoc condotta su 1342 pazienti, l'effetto del losartan sull'AU spiegava circa il $20 \%$ della sua azione nefroprotettiva (41). Questi dati confermano e ampliano quelli derivanti dallo studio LIFE che segnalavano una relazione lineare tra la riduzione dell'AU e quella degli eventi CV nei pazienti sotto losartan. L'effetto protettivo in termini CV e renali che si è osservato potrebbe essere, almeno in parte, dovuto all'azione uricosurica del losartan. Infatti, questa molecola aumenta l'escrezione urinaria di urato inibendo l'assorbimento renale mediato dell'URAT-1. Questo trasportatore di anioni organici è stato recentemente descritto non solo a livello delle cellule tubulari prossimali ma anche in cellule muscolari lisce di aorta umana (42). Si potrebbe, pertanto, speculare sul fatto che il losartan possa attuare il suo effetto protettivo prevenendo l'entrata dell'AU nelle cellule muscolari lisce vascolari, riducendo così la proliferazione cellulare e l'infiltrato infiammatorio mediato dall'espressione di MCP-1. Ulteriori studi con un disegno prospettico sono necessari per confermare questi dati incoraggianti.

\section{Conclusioni}

Numerosi studi suggeriscono che l'iperuricemia anche lieve sia da considerarsi un predittore indipendente di eventi CV e renali e che possa essere implicata nello sviluppo iniziale del danno d'organo. Sebbene siano disponibili dati preliminari che fanno ipotizzare che la riduzione farmacologica dei livelli di acido urico possa contribuire alla protezione $\mathrm{CV}$ e renale, sono necessari studi randomizzati condotti su larga scala per poter confermare il ruolo dell'AU come potenziale target della terapia cardio-renale.

\section{Riassunto}

Numerosi studi epidemiologici sottolineano una relazione tra livelli di acido urico e condizioni cliniche quali ipertensione, insulino-resistenza e malattia renale e cardiovascolare. Infatti, con la rapida diffusione di tali condizioni, si è assistito al progressivo aumento dei valori medi di acido urico nella popolazione generale (da $3.5 \mathrm{mg} / \mathrm{dL}$ negli anni '20 a $6.5 \mathrm{mg} / \mathrm{dL}$ negli anni ' 70 ).

In questa revisione cercheremo di approfondire se l'acido urico possa essere considerato un promotore attivo del danno aterosclerotico o solo un marcatore indipendente di un aumentato rischio di malattia. Nonostante non vi sia ancora un'indicazione basata sulle evidenze al trattamento farmacologico dell'iperuricemia asintomatica, recenti studi suggeriscono una relazione diretta tra le variazioni di $\mathrm{AU}$ indotte dalla terapia e l'incidenza di eventi cardio-renali. 
Parole chiave: Acido urico, Danno d'organo, Malattia renale cronica, Rischio cardiovascolare

Dichiarazione di conflitto di interessi: Gli Autori dichiarano di non avere conflitto di interessi.

Indirizzo degli Autori:

Dr.ssa Francesca Viazzi

Dipartimento di Cardionefrologia, Università di Genova

IRCCS Azienda Ospedaliera Universitaria San Martino-IST

Istituto Nazionale per la ricerca sul Cancro

Viale Benedetto XV 6

16132 Genova

francesca.viazzi@unige.it

\section{Bibliografia}

1. Choi HK, Curhan G. Independent impact of gout on mortality and risk for coronary heart disease. Circulation 2007; 21 (116): 894-900.

2. Feig DI, Kang DH, Johnson RJ. Uric acid and cardiovascular risk. N Engl J Med 2008; 359: 1811-21.

3. Mahomed FA. On chronic Bright's disease, and its essential symptoms. Lancet 1879; 1: 399-401.

4. Lin CS, Lee WL, Hung YJ, et al. Prevalence of hyperuricemia and its association with antihypertensive treatment in hypertensive patients in Taiwan. Int J Cardiol 2012; 156: 41-6.

5. Grayson PC, Kim SY, LaValley M, Choi HK. Hyperuricemia and incident hypertension: a systematic review and metaanalysis. Arthritis Care Res (Hoboken). 2011; 63: 102-10.

6. Feig DI. Uric acid and hypertension. Semin Nephrol 2011; 31 : 441-6.

7. Feig DI, Soletsky B, Johnson RJ. Effect of allopurinol on blood pressure of adolescents with newly diagnosed essential hypertension: a randomized trial. JAMA 2008; 300: 924-32.

8. Soletsky B, Feig D. Uric Acid Reduction Rectifies Prehypertension in Obese Adolescents. Hypertension 2012; 60: 1148-56.

9. Lin SD, Tsai DH, Hsu SR. Association between serum uric acid level and components of the metabolic syndrome. J Chin Med Assoc 2006; 69: 512-6.

10. Kodama S, Saito K, Yachi Y, et al. Association between serum uric acid and development of type 2 diabetes. Diabetes Care 2009; 32: 1737-42.

11. Viazzi F, Leoncini G, Vercelli M, Deferrari G, Pontremoli R. Serum uric acid levels predict new-onset type 2 diabetes in hospitalized patients with primary hypertension: the MAGIC study. Diabetes Care 2011; 34: 126-8.

12. Sánchez-Lozada LG, Tapia E, Bautista-García P, et al. Effects of febuxostat on metabolic and renal alterations in rats with fructose-induced metabolic syndrome. Am J Physiol Renal Physiol 2008; 294: F710-8.

13. Feig DI, Kang DH, Nakagawa T, Mazzali M, Johnson RJ. Uric acid and hypertension. Curr Hypertens Rep 2006; 8: 111-5.

14. Kanellis $\mathrm{J}$, Watanabe $\mathrm{S}$, Li JH, et al. Uric acid stimulates monocyte chemoattractant protein-1 production in vascular smooth muscle cells via mitogen-activated protein kinase and cyclooxygenase-2. Hypertension 2003; 41: 1287-93.

15. Cuspidi C, Valerio C, Sala C, et al. Lack of association between serum uric acid and organ damage in a never-treated essential hypertensive population at low prevalence of hyperuricemia. Am J Hypertens 2007; 20: 678-85.

16. Viazzi F, Parodi D, Leoncini G, et al. Serum uric acid and target organ damage in primary hypertension. Hypertension 2005; 45 : 991-6.

17. Tuttle KR, Short RA, Johnson RJ. Sex differences in uric acid and risk factors for coronary artery disease. Am J Cardiol 2001; 87: $1411-4$

18. Krishnan E, Pandya BJ, Chung L, Dabbous O. Hyperuricemia and the risk for subclinical coronary atherosclerosis - data from a prospective observational cohort study. Arthritis Res Ther 2011; 13: R66.

19. Iwashima Y, Horio T, Kamide K, Rakugi H, Ogihara T, Kawano Y. Uric acid, left ventricular mass index, and risk of cardiovascular disease in essential hypertension. Hypertension 2006; 47: 195-202.

20. Culleton BF, Larson MG, Kannel WB, Levy D. Serum uric acid and risk for cardiovascular disease and death: the Framingham Heart Study. Ann Intern Med 1999; 131: 7-13.

21. Wannamethee SG, Shaper AG, Whincup PH. Serum urate and the risk of major coronary heart disease events. Heart 1997; 78: 147-53.

22. Høieggen A, Alderman MH, Kjeldsen SE, et al. The impact of serum uric acid on cardiovascular outcomes in the LIFE study. Kidney Int 2004; 65: 1041-9.

23. Bos MJ, Koudstaal PJ, Hofman A, Witteman JC, Breteler MM. Uric acid is a risk factor for myocardial infarction and stroke: the Rotterdam study. Stroke 2006; 37: 1503-7.

24. Fang J, Alderman MH. Serum uric acid and cardiovascular mortality: the NHANES I epidemiologic follow-up study, 1971-1992. National Health and Nutrition Examination Survey. JAMA 2000; 283: 2404-10.

25. Krishnan E, Baker JF, Furst DE, Schumacher HR. Gout and the risk of acute myocardial infarction. Arthritis Rheum 2006; 54 : 2688-96.

26. Kim SY, Guevara JP, Kim KM, Choi HK, Heitjan DF, Albert DA. Hyperuricemia and coronary heart disease: a systematic review and meta-analysis. Arthritis Care Res (Hoboken) 2010; 62: 170-80.

27. Kim SY, Guevara JP, Kim KM, Choi HK, Heitjan DF, Albert DA. Hyperuricemia and risk of stroke: a systematic review and meta-analysis. Arthritis Rheum 2009; 61: 885-92.

28. Hsu SP, Pai MF, Peng YS, Chiang CK, Ho TI, Hung KY. Serum uric acid levels show a 'J-shaped' association with all-cause mortality in haemodialysis patients. Nephrol Dial Transplant 2004; 19: 457-62.

29. Neri L, Rocca Rey LA, Lentine KL, et al. Joint association of hyperuricemia and reduced GFR on cardiovascular morbidity: a historical cohort study based on laboratory and claims data from a national insurance provider. Am J Kidney Dis 2011; 58: 398-408.

30. Latif W, Karaboyas A, Tong L, et al. Uric acid levels and allcause and cardiovascular mortality in the hemodialysis population. Clin J Am Soc Nephrol 2011; 6: 2470-7. 
31. Lin CS, Hung YJ, Chen GY, et al. A multicenter study of the association of serum uric acid, serum creatinine, and diuretic use in hypertensive patients. Int J Cardiol 2011; 148: 325-30.

32. Viazzi F, Leoncini G, Ratto E, et al. Mild hyperuricemia and subclinical renal damage in untreated primary hypertension. Am J Hypertens 2007; 20: 1276-82.

33. Ishani A, Grandits GA, Grimm RH, et al. Association of single measurements of dipstick proteinuria, estimated glomerular filtration rate, and hematocrit with 25- year incidence of endstage renal disease in the multiple risk factor intervention trial. J Am Soc Nephrol 2006; 17: 1444-52.

34. Obermayr RP, Temml C, Gutjahr G, Knechtelsdorfer M, Oberbauer R, Klauser-Braun R. Elevated uric acid increases the risk for kidney disease. J Am Soc Nephrol 2008; 19: 2407-13.

35. Weiner DE, Tighiouart H, Elsayed EF, Griffith JL, Salem DN, Levey AS. Uric acid and incident kidney disease in the community. J Am Soc Nephrol 2008; 19: 1204-11.

36. Malheiro J, Almeida M, Fonseca I, et al. Hyperuricemia in adult renal allograft recipients: prevalence and predictors. Transplant Proc 2012; 44: 2369-72.

37. Huang Y, Li YL, Huang H, Wang L, Yuan WM, Li J. Effects of hyperuricemia on renal function of renal transplant recipients: a systematic review and meta-analysis of cohort studies. PLoS One 2012; 7 (6): e39457.

38. Kanbay M, Ozkara A, Selcoki Y, et al. Effect of treatment of hyperuricemia with allopurinol on blood pressure, creatinine clearence, and proteinuria in patients with normal renal functions. Int Urol Nephrol 2007; 39: 1227-33.

39. Luk AJ, Levin GP, Moore EE, Zhou XH, Kestenbaum BR, Choi HK. Allopurinol and mortality in hyperuricaemic patients. Rheumatology (Oxford) 2009; 48: 804-6.

40. Goicoechea M, de Vinuesa SG, Verdalles U, et al. Effect of allopurinol in chronic kidney disease progression and cardiovascular risk. Clin J Am Soc Nephrol 2010; 5: 1388-93.

41. Miao Y, Ottenbros SA, Laverman GD, et al. Effect of a reduction in uric acid on renal outcomes during losartan treatment: a post hoc analysis of the reduction of endpoints in non-insulindependent diabetes mellitus with the Angiotensin II Antagonist Losartan Trial. Hypertension 2011; 58: 2-7.

42. Price KL, Sautin YY, Long DA, et al. Human vascular smooth muscle cells express a urate transporter. J Am Soc Nephrol 2006; 17: 1791-5. 